\title{
Molecular genetics of familial cerebral cavernous malformations
}

\author{
Shervin R. Dashti, M.D., Ph.D., Alan Hoffer, M.D., Yin C. Hu, M.D., \\ AND WarRen R. SElman, M.D.
} Department of Neurosurgery, University Hospitals of Cleveland, Case School of Medicine,
Cleveland, Ohio

\begin{abstract}
$\checkmark$ Cerebral cavernous malformations (CMs) are angiographically occult neurovascular lesions that consist of enlarged vascular channels without intervening normal parenchyma. Cavernous malformations can occur as sporadic or autosomal-dominant inherited conditions. Approximately 50\% of Hispanic patients with cerebral CMs have the familial form, compared with 10 to $20 \%$ of Caucasian patients. There is no difference in the pathological findings or presentation in the sporadic and familial forms. To date, familial CMs have been attributed to mutations at three different loci: $C C M 1$ on 7q21.2, CCM2 on 7p15-p13, or $C C M 3$ on 3q25.2-q27. The authors summarize the current understanding of the molecular events underlying familial CMs.
\end{abstract}

\section{KEY WoRDS - cavernoma - cerebral cavernous malformation • KRIT1 gene • CCM gene}

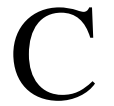

EREBRAL CMs, or cavernomas, are benign vascular hamartomas that consist of endothelium-lined vascular channels without intervening normal brain parenchyma. The prevalence of CMs in the general population has been estimated at 0.1 to $0.5 \%$ based on large autopsy studies.22 Clinically, cerebral CMs usually present with seizures or hemorrhage. The natural history of symptomatic CMs involves repeated hemorrhages, with a gradual increase in the size and number of the vascular channels.

On neuroimaging, cavernomas may be undetectable by computed tomography scanning in up to 30 to $50 \%$ of cases. When visualized, they appear as rounded, hyperdense lesions with or without calcium. The classic appearance on magnetic resonance imaging is a "popcorn ball" consisting of a reticulated, heterogeneous core surrounded by a hypointense hemosiderin rim on $\mathrm{T}_{2}$-weighted images. The $\mathrm{T}_{2}$-weighted gradient echo images demonstrate a prominent susceptibility effect (blooming) and are the most sensitive diagnostic sequence. Fluid-attenuated inversionrecovery and $\mathrm{T}_{2}$-weighted magnetic resonance images may show surrounding edema in lesions with acute hemorrhage.

\footnotetext{
Abbreviations used in this paper: $\mathrm{CM}=$ cavernous malformation; $\mathrm{COX}-2$ = cyclooxygenase- 2 ; $\mathrm{ECM}=$ extracellular matrix; GTPase $=$ guanosine $5^{\prime}$-triphosphatase ICAP $=$ integrin cytoplasmic domain-associated protein; KRIT $=$ Krev interaction trapped; MAPK $=$ mitogen-activated protein kinase; MEKK3 = MAPK kinase kinase 3; MEK1/2-ERK 1/2 = MAPK kinase 1/2-extracellular signal regulated protein kinase $1 / 2 ; \mathrm{OSM}=$ osmosensing scaffold for MEKK3.
}

Although CMs are angiographically occult, associated anomalies such as venous angiomas may be visualized.

Cerebral CMs can occur sporadically or as an autosomaldominant inherited condition. Approximately 50\% of Hispanic patients with a cerebral CM have the familial form, compared with 10 to $20 \%$ of Caucasian patients. ${ }^{7,24}$ The familial form of cerebral CM usually presents with multiple cavernomas, in contrast to sporadic cases, in which the lesions are usually solitary. ${ }^{18,24}$ There is no difference in the pathological features or clinical presentation of the sporadic and familial forms.

The exact mechanism of familial CM pathogenesis is still unknown. To date, familial forms of the disease have been attributed to mutations at three different loci: $C C M 1$ on 7q21.2, CCM2 on 7p15-p13, or CCM3 on 3q25.2-q27. Multilocus analysis of familial CMs shows $40 \%$ of kindred linked to the $C C M 1$ locus, $20 \%$ linked to $C C M 2$, and $40 \%$ linked to $C C M 3{ }^{6}$

\section{Discussion}

\section{The CCM1 Locus (KRIT1 Gene)}

The CCM1 locus is mutated in nearly all Hispanic-American patients as well as in approximately $40 \%$ of all Caucasian patients with familial cavernomas. ${ }^{6,14,18}$ Although familial and sporadic CMs are phenotypically identical, no mutation in the CCMI locus has been found in sporadic cases in patients harboring single $\mathrm{CMs}{ }^{23}$ The $C C M 1$ locus has been identified as the KRIT-1 gene located on 7q21.2; Krev-1 is a conserved Ras-family GTPase with unknown cellular function. 
The protein KRIT-1, the product of the KRIT- 1 gene, has been found in vascular endothelium, astrocytes, and pyramidal cells of the adult brain. ${ }^{15}$ The essential function of this protein is demonstrated in KRIT- 1 knockout mice, which die at midgestation of vascular pathology. ${ }^{29}$ These mice demonstrate marked enlargement of and increased endothelial proliferation in the dorsal aorta, defects that are associated with early downregulation of artery-specific genes.

The KRIT-1 protein has also been shown to associate with the integrin-binding protein ICAP $1 \alpha>{ }^{31}$ Integrin molecules are transmembrane receptor proteins that play a critical role in endothelial cell-cell and cell-ECM interactions as well as in endothelial cell migration and lumen formation during angiogenesis. ${ }^{2-4,9}$ The cytoplasmic portion of integrin proteins lacks inherent enzymatic activity and relies on linker proteins for connection to the actin cytoskeleton and for downstream intracellular signaling.,3 The ICAP $1 \alpha$, a broadly distributed 200 amino acid protein, has been identified through yeast hybrid assays to bind avidly to the $\beta 1$ integrin cytoplasmic domain..$^{33}$ The integrin-binding site is located at the amino terminus of the KRIT-1 protein. Mutations of this motif result in complete loss of KRIT-1/ICAP1 $\alpha$ interaction. ${ }^{31}$ Most mutations in the KRIT1 gene responsible for familial CM result in early stop codons and truncated protein synthesis, hinting at a defect in ICAP1 $\alpha$-mediated ECM interaction as a possible contributor to CM pathogenesis. Interestingly, results of competition assays support the suggestion that KRIT- 1 and $\beta 1$ integrin compete for the same binding site on ICAP1 $\alpha$, suggesting a possible regulatory mechanism. ${ }^{33}$ In the $C C M 1$ locus, KRIT-1 protein is truncated and lacks the integrin-binding site, which would shift the system toward ICAP $1 \alpha$-integrin interaction.

Gunel, et al., ${ }^{14}$ have demonstrated through immunohistochemical and coimmunoprecipitation studies that KRIT-1 protein localizes to microtubules. Specifically, KRIT-1 colocalizes with microtubule plus ends during the late phase of mitosis. These authors' results show that KRIT-1 is a microtubule-associated protein, possibly responsible for microtubule targeting. Furthermore, interaction of KRIT-1 with Krev1 and ICAP1 $\alpha$-integrin suggests that KRIT-1 may help modulate the cytoskeleton, and thus shape endothelial cell morphology and function in response to cellmatrix and cell-cell interactions. Gunel, et al., ${ }^{14}$ propose that the loss of this targeting mechanism would lead to abnormal endothelial tube development and the subsequent appearance of cerebral CMs.

\section{The CCM2 Locus (MGC4607 or malcaverin Gene)}

The $C C M 2$ locus has been identified as the MGC4607 or malcaverin gene located on $7 \mathrm{p} 15$-p13. It encodes a protein containing a putative phosphotyrosine-binding domain. ${ }^{19}$ Although protein sequencing fails to show any known conserved regions that might define the function of the CCM2 gene, a clue may come from the CCM2 mouse homolog, the OSM. The OSM is involved in the cellular response to osmotic insults, and is required for MEKK3-mediated activation of p38 in response to cellular stress. ${ }^{27}$ It is known that $\mathrm{p} 38$, a member of the MAPKs, is one of several intracellular kinases that transduce signals essential for vascular remodeling and maturation. ${ }^{16,17,26}$ The p38 kinase negative- ly regulates endothelial cell survival, proliferation, and differentiation in fibroblast growth factor 2-stimulated angiogenesis..$^{20}$ This kinase is also an important mediator of vascular endothelial growth factor activation of the COX-2 expression in endothelial cells during angiogenesis. ${ }^{30}$ The p38 MAPK also mediates tumor necrosis factor-induced apoptosis in endothelial cells through two known mechanisms: 1) phosphorylation and downregulation of the antiapoptotic protein $\mathrm{Bcl}-\mathrm{xL}$; and 2) phosphorylation of the proapoptotic protein Bad through suppression of the MEK1/2-ERK1/2 survival pathway (Fig. 1). ${ }^{10,11}$ McMullen and colleagues ${ }^{21}$ demonstrated cell cycle arrest in endothelial cells transfected with an upstream activator of $\mathrm{p} 38$ MAPK. The same cells, however, demonstrated increased migration, with alterations in the actin ultrastructure and enhanced lamellipodia. Further evidence for a regulatory capacity for p38 in angiogenesis is provided by its critical role in mural cell recruitment during neovascularization in the rat aorta model of angiogenesis. ${ }^{34}$

Using in situ hybridization, Seker, et al. ${ }^{25}$ found significant colocalization of CCM2 with CCM1 messenger RNA in embryonal and postnatal mice. This was confirmed at the protein expression level, with both proteins present in arterial endothelium as well as in pyramidal neurons and the foot processes of astrocytes. Coimmunoprecipitation and fluorescence resonance energy transfer studies showed that the CCM1 and CCM2 gene products interact with each other. ${ }^{32}$ In much the same way that CCM1 and $\beta 1$ integrin interact with ICAP1 $\alpha$, this interaction is dependent on the phosphotyrosine-binding domain of CCM2 and is inhibited by a familial CCM2 missense mutation, suggesting that loss of this interaction may be another step in the pathogenesis of familial cavernomas. ${ }^{32}$ The parallel expression and mutual binding of CCM1 and CCM2 suggests that they may function through the same regulatory pathways in cavernoma formation (Fig. 2). Moreover, CCM1, CCM2, and MEKK3 bind in a ternary complex, suggesting that the pathways are not simply parallel but probably converge.

In the cell, the OSM localizes to Rac-containing membrane ruffles ${ }^{27}$ (Rac is a Rho-GTPase that is known to regulate endothelial motility and morphology during angiogenesis). ${ }^{8}$ The ICAP1 $\alpha$, which binds KRIT-1, is also a known inhibitor of Rac signaling. Modulation of Rac-mediated angiogenesis may represent another potential point of convergence in action for CCM1 and CCM2 (Fig. 2). ${ }^{25}$

\section{The CCM3 Locus (PCD10 Gene)}

Approximately $40 \%$ of kindred with familial CMs show linkage to the $C C M 3$ locus. ${ }^{6}$ The $C C M 3$ locus has been identified as the PCD10 gene located on 3q25.2-27. ${ }^{1}$ The PCD10 gene codes for a 212 amino acid protein lacking any known domains. This protein has been linked to apoptosis, which is an essential process in arterial morphogenesis. ${ }^{5}$ Interestingly, apoptosis in smooth-muscle cells has been shown to be mediated by a $\beta 1$ integrin signaling cascade, providing a possible link with $C C M 1$ and CCM2 in the formation of cerebral CMs. ${ }^{28}$

\section{Conclusions}

The exact mechanism of familial CM pathogenesis is 
Molecular genetics of familial cerebral cavernous malformations

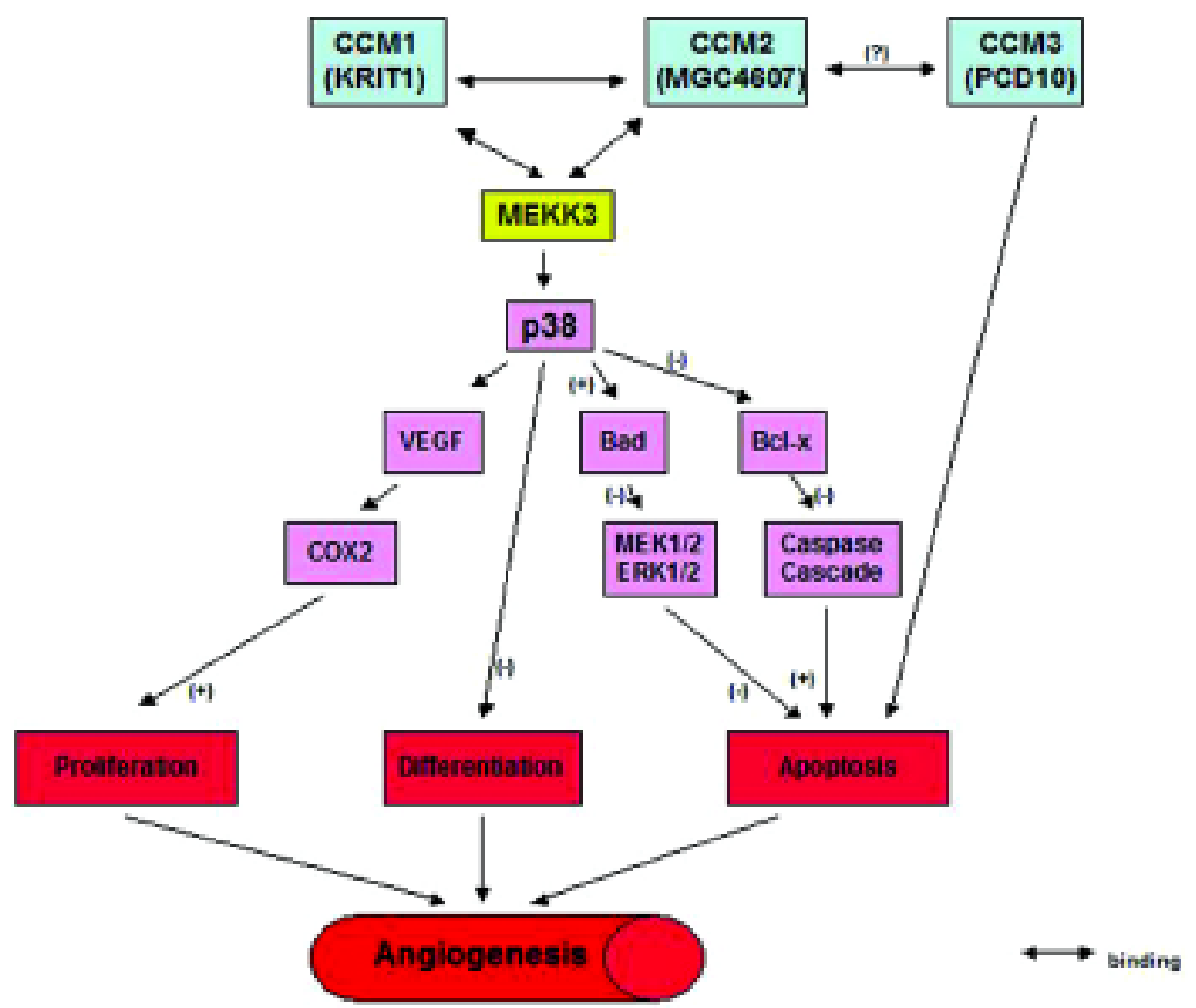

FIG. 1. Flow chart summarizing the role of the p38 MAPK pathway in angiogenesis and cavernoma formation. Gene names are in parentheses. VEGF = vascular endothelial growth factor.

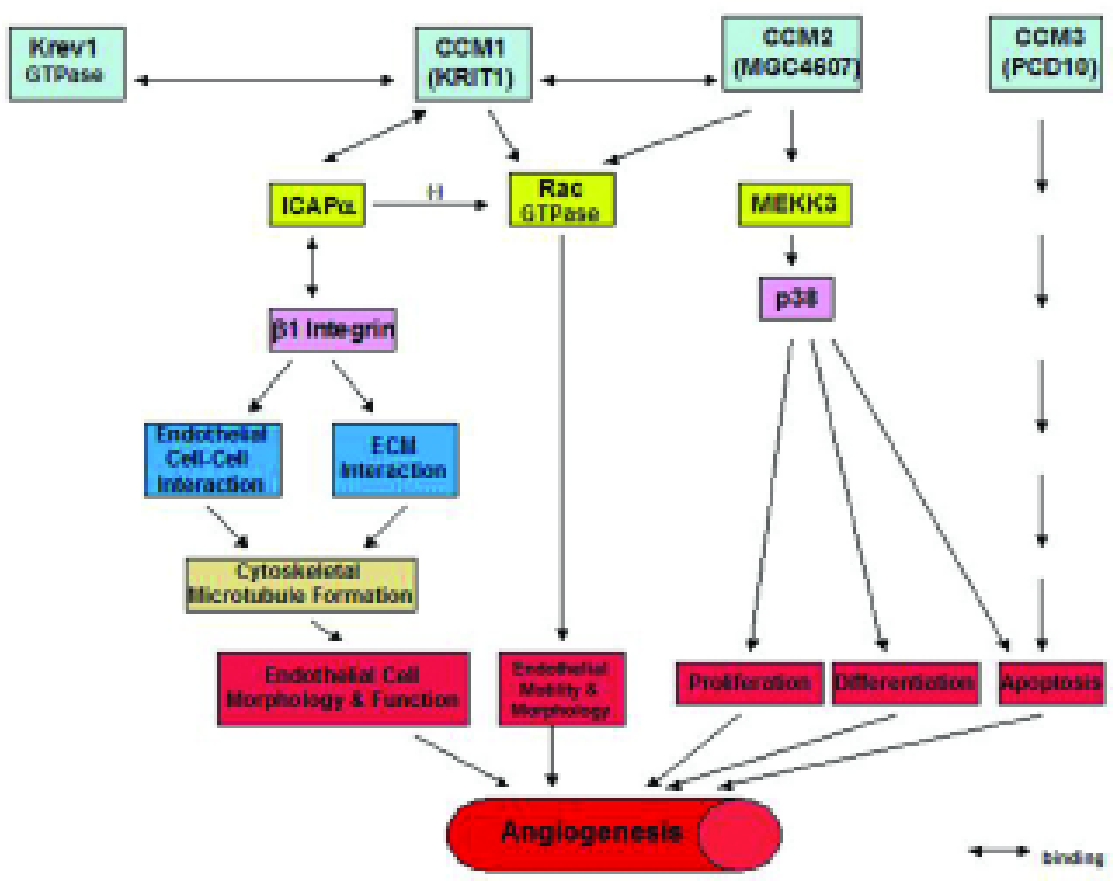

FIG. 2. Flow chart summarizing the signaling pathways in familial CMs. 
still unknown. Mutation at three distinct loci (CCMI, $C C M 2$, and $C C M 3$ ) has been shown in cases of familial cerebral CM. Because familial cavernomas caused by different gene mutations are pathologically and phenotypically indistinguishable, it has been postulated that the three $C C M$ genes act through the same molecular pathway. ${ }^{12,13,25}$ There is growing evidence that $C C M 1$ may play a role in regulating $\beta 1$ integrin-mediated angiogenesis through KRIT-1, which is involved with a bidirectional signaling pathway between the ECM and the cytoskeleton that uses an integrin-mediated cascade. ${ }^{25}$ Future studies detailing this signaling cascade will shed new light on cerebral CM pathogenesis.

The p38 MAPK is a ubiquitously expressed intracellular kinase that regulates endothelial cell survival, proliferation, and differentiation. ${ }^{20}$ Regulation of the p38 MAPK cascade may be one of the final common pathways for modulation of angiogenesis by the $C C M$ genes. The CCM1 and CCM2 gene products influence the $\mathrm{p} 38$ MAPK pathway by activating its upstream kinase (MEKK3). Immunohistochemical and binding studies could be used to investigate whether the CCM3 protein also participates in the interaction required for MEKK3 and p38 activation. Any interaction of the CCM gene products with MEKK6, the other upstream regulator of $\mathrm{p} 38$, needs to be clarified. Cross-talk of p38 with other members of the MAPK pathway, such as MEK/ERK, needs further elucidation as well.

Development of dominant negative $C C M$ genes, if possible, would be valuable for future studies. Endothelial cell lines could then be transfected with the dominant negative $C C M$ genes, and the activated phospho-p38 MAPK levels, as well as apoptotic or differentiation markers, could be assayed with Western blot analysis. The convergence of $C C M 2$ and $C C M 3$ on the apoptotic mechanisms is yet another area that requires further study.

It has been shown that $C C M 1$ knockout mice die in utero. Future development of $C C M 2$ or $C C M 3$ knockout mice, especially if they were to survive, would also allow better characterization of the intracellular signaling pathways, including p38 MAPK. Finally, modulation of Racmediated angiogenesis may represent another potential point of convergence in action for the $C C M$ genes that warrants further study (Fig. 2). ${ }^{25}$

\section{References}

1. Bergametti F, Denier C, Labauge P, Arnoult M, Boetto S, Clanet M, et al: Mutations within the programmed cell death 10 gene cause cerebral cavernous malformations. Am J Hum Genet 76:42-51, 2005

2. Bloch W, Forsberg E, Lentini S, Brakebusch C, Martin K, Krell $\mathrm{HW}$, et al: Beta 1 integrin is essential for teratoma growth and angiogenesis. J Cell Biol 139:265-278, 1997

3. Brooks PC: Cell adhesion molecules in angiogenesis. Cancer Metastasis Rev 15:187-194, 1996

4. Brooks PC: Role of integrins in angiogenesis. Eur J Cancer 32A:2423-2429, 1996

5. Busch CR, Heath DD, Hubberstey A: Sensitive genetic biomarkers for determining apoptosis in the brown bullhead (Ameiurus nebulosus). Gene 329:1-10, 2004

6. Craig HD, Gunel M, Cepeda O, Johnson EW, Ptacek L, Steinberg GK, et al: Multilocus linkage identifies two new loci for a mendelian form of stroke, cerebral cavernous malformation, at 7p15-13 and 3q25.2-27. Hum Mol Genet 7: 1851-1858, 1998
7. Denier C, Goutagny S, Labauge P, Krivosic V, Arnoult M, Cousin A, et al: Mutations within the MGC4607 gene cause cerebral cavernous malformations. Am J Hum Genet 74: 326-337, 2004

8. Fryer BH, Field J: Rho, Rac, Pak and angiogenesis: old roles and newly identified responsibilities in endothelial cells. Cancer Lett 229:13-23, 2005

9. Gamble J, Meyer G, Noack L, Furze J, Matthias L, Kovach N, et al: B1 integrin activation inhibits in vitro tube formation: effects on cell migration, vacuole coalescence and lumen formation. Endothelium 7:23-34, 1999

10. Grethe S, Ares MP, Andersson T, Porn-Ares MI: p38 MAPK mediates TNF-induced apoptosis in endothelial cells via phosphorylation and downregulation of Bcl- $\mathrm{x}_{\mathrm{L}}$. Exp Cell Res 298: 632-642, 2004

11. Grethe S, Porn-Ares MI: p38 MAPK regulates phosphorylation of Bad via PP2A-dependent suppression of the MEK1/2ERK1/2 survival pathway in TNF- $\alpha$ induced endothelial apoptosis. Cell Signal 18:531-540, 2006

12. Gunel M, Awad IA, Finberg K, Anson JA, Steinberg GK, Batjer $\mathrm{HH}$, et al: A founder mutation as a cause of cerebral cavernous malformation in Hispanic Americans. N Engl J Med 334: 946-951, 1996

13. Gunel M, Awad IA, Finberg K, Steinberg GK, Craig HD, Cepeda O, et al: Genetic heterogeneity of inherited cerebral cavernous malformation. Neurosurgery 38:1265-1271, 1996

14. Gunel M, Laurans MS, Shin D, DiLuna ML, Voorhees J, Choate K, et al: KRIT1, a gene mutated in cerebral cavernous malformation, encodes a microtubule-associated protein. Proc Natl Acad Sci U S A 99:10677-10682, 2002

15. Guzeloglu-Kayisli O, Amankulor NM, Voorhees J, Luleci G, Lifton RP, Gunel M: KRIT1/cerebral cavernous malformation 1 protein localizes to vascular endothelium, astrocytes, and pyramidal cells of the adult human cerebral cortex. Neurosurgery 54:943-949, 2004

16. Issbrucker $\mathrm{K}$, Marti HH, Hippenstiel S, Springmann G, Voswinckel R, Gaumann A, et al: p38 MAP kinase-a molecular switch between VEGF-induced angiogenesis and vascular hyperpermeability. FASEB J 17:262-264, 2003

17. Jackson JR, Bolognese B, Hillegass L, Kassis S, Adams J, Griswold DE, et al: Pharmacological effects of SB 220025, a selective inhibitor of P38 mitogen-activated protein kinase, in angiogenesis and chronic inflammatory disease models. J Pharmacol Exp Ther 284:687-692, 1998

18. Labauge P, Laberge S, Brunereau L, Levy C, Tournier-Lasserve E: Hereditary cerebral cavernous angiomas: Clinical and genetic features in 57 French families. Societe Française de Neurochirurgie. Lancet 352:1892-1897, 1998

19. Liquori CL, Berg MJ, Siegel AM, Huang E, Zawistowski JS, Stoffer T, et al: Mutations in a gene encoding a novel protein containing a phosphotyrosine-binding domain cause type 2 cerebral cavernous malformations. Am J Hum Genet 73: 1459-1464, 2003

20. Matsumoto T, Turesson I, Book M, Gerwins P, Claesson-Welsh L: p38 MAP kinase negatively regulates endothelial cell survival, proliferation, and differentiation in FGF-2-stimulated angiogenesis. J Cell Biol 156:149-160, 2002

21. McMullen ME, Bryant PW, Glembotski CC, Vincent PA, Pumiglia KM: Activation of p38 has opposing effects on the proliferation and migration of endothelial cells. J Biol Chem 280: 20995-21003, 2005

22. Otten P, Pizzolato GP, Rilliet B, Berney J: [131 cases of cavernous angioma (cavernomas) of the CNS, discovered by retrospective analysis of 24,535 autopsies.] Neurochirurgie 35: 82-83, 128-131, 1989 (Fr)

23. Reich P, Winkler J, Straube A, Steiger HJ, Peraud A: Molecular genetic investigations in the CCM1 gene in sporadic cerebral cavernomas. Neurology 60:1135-1138, 2003

24. Rigamonti D, Hadley MN, Drayer BP, Johnson PC, Hoenig- 


\section{Molecular genetics of familial cerebral cavernous malformations}

Rigamonti K, Knight JT, et al: Cerebral cavernous malformations. Incidence and familial occurrence. N Engl J Med 319:343-347, 1988

25. Seker A, Pricola KL, Guclu B, Ozturk AK, Louvi A, Gunel M: CCM2 expression parallels that of CCM1. Stroke 37:518-523, 2006

26. Tanaka K, Abe M, Sato Y: Roles of extracellular signal-regulated kinase $1 / 2$ and p38 mitogen-activated protein kinase in the signal transduction of basic fibroblast growth factor in endothelial cells during angiogenesis. Jpn J Cancer Res 90:647-654, 1999

27. Uhlik MT, Abell AN, Johnson NL, Sun W, Cuevas BD, LobelRice KE, et al: Rac-MEKK3-MKK3 scaffolding for p38 MAPK activation during hyperosmotic shock. Nat Cell Biol 5:1104-1110, 2003

28. Wernig F, Mayr M, Xu Q: Mechanical stretch-induced apoptosis in smooth muscle cells is mediated by $\beta_{1}$-integrin signaling pathways. Hypertension 41:903-911, 2003

29. Whitehead KJ, Plummer NW, Adams JA, Marchuk DA, Li DY: $\mathrm{Ccm} 1$ is required for arterial morphogenesis: implications for the etiology of human cavernous malformations. Development 131:1437-1448, 2004

30. Wu G, Luo J, Rana JS, Laham R, Sellke FW, Li J: Involvement of COX-2 in VEGF-induced angiogenesis via P38 and JNK pathways in vascular endothelial cells. Cardiovasc Res 69:512-519, 2006

31. Zawistowski JS, Serebriiskii IG, Lee MF, Golemis EA,
Marchuk DA: KRIT1 association with the integrin-binding protein ICAP-1: a new direction in the elucidation of cerebral cavernous malformations (CCM1) pathogenesis. Hum Mol Genet 11:389-396, 2002

32. Zawistowski JS, Stalheim L, Uhlik MT, Abell AN, Ancrile BB, Johnson GL, et al: CCM1 and CCM2 protein interactions in cell signaling: implications for cerebral cavernous malformations pathogenesis. Hum Mol Genet 14:2521-2531, 2005

33. Zhang J, Clatterbuck RE, Rigamonti D, Chang DD, Dietz HC: Interaction between krit 1 and icap $1 \alpha$ infers perturbation of integrin $\beta 1$-mediated angiogenesis in the pathogenesis of cerebral cavernous malformation. Hum Mol Genet 10:2953-2960, 2001

34. Zhu WH, Han J, Nicosia RF: Requisite role of p38 MAPK in mural cell recruitment during angiogenesis in the rat aorta model. J Vasc Res 40:140-148, 2003

Manuscript received April 18, 2006.

Accepted in final form June 1, 2006.

Address reprint requests to: Warren R. Selman, M.D., Department of Neurosurgery, University Hospitals of Cleveland, 11100 Euclid Avenue, Cleveland, Ohio 44106. email: warren.selman@ uhhs.com. 